\title{
WAVES BELOW FIRST CUTOFF IN A DUCT
}

\author{
RODNEY F. DAVIDSON ${ }^{i}$
}

(Received 11 December 1986; revised 9 February 1987)

\begin{abstract}
The two-dimensional Helmholtz equation is studied for an infinite region with two semi-infinite plates extending to infinity in opposite directions and a finite duct in the overlapping region. The solution technique leads to coupled Wiener-Hopf equations, and subsequently to an infinite set of simultaneous linear equations. As an example, an asymptotic expansion is calculated and graphed for the case when the duct length divided by duct width is large enough to ensure damping of all but the zero mode wave in the duct.
\end{abstract}

\section{Introduction}

The problem of wave fields in infinite regions penetrating apertures and waveguides has been studied extensively. Generally, the method of solution relies on solving in various regions, and linking these solutions across connecting boundaries. Further, if edges are present, edge conditions must be obeyed to ensure uniqueness [2].

This paper investigates the steady state two-dimensional problem of the fields due to a unit source in a boundary configuration as shown in Figure 1. Problems similar to this one have been discussed by Noble in his book on the Wiener-Hopf technique [6].

The fields are solved in each of the regions $y<d_{2}, d_{2}<y<d_{1}$ and $y>d_{1}$, and linked across the connecting boundaries. This linking leads to equations that are able to be solved by the Wiener-Hopf technique. However, the problems solved in Noble's book have both plates extending to infinity on the same side. This problem has the plates extending to infinity on opposite sides, and a finite waveguide formed between the plates. For this problem, the conditions $a_{1}>a_{2}$

${ }^{1}$ P. O. Box 366, Mentone, Victoria 3194, Australia.

(C) Copyright Australian Mathematical Society 1988, Serial-fee code 0334-2700/88 


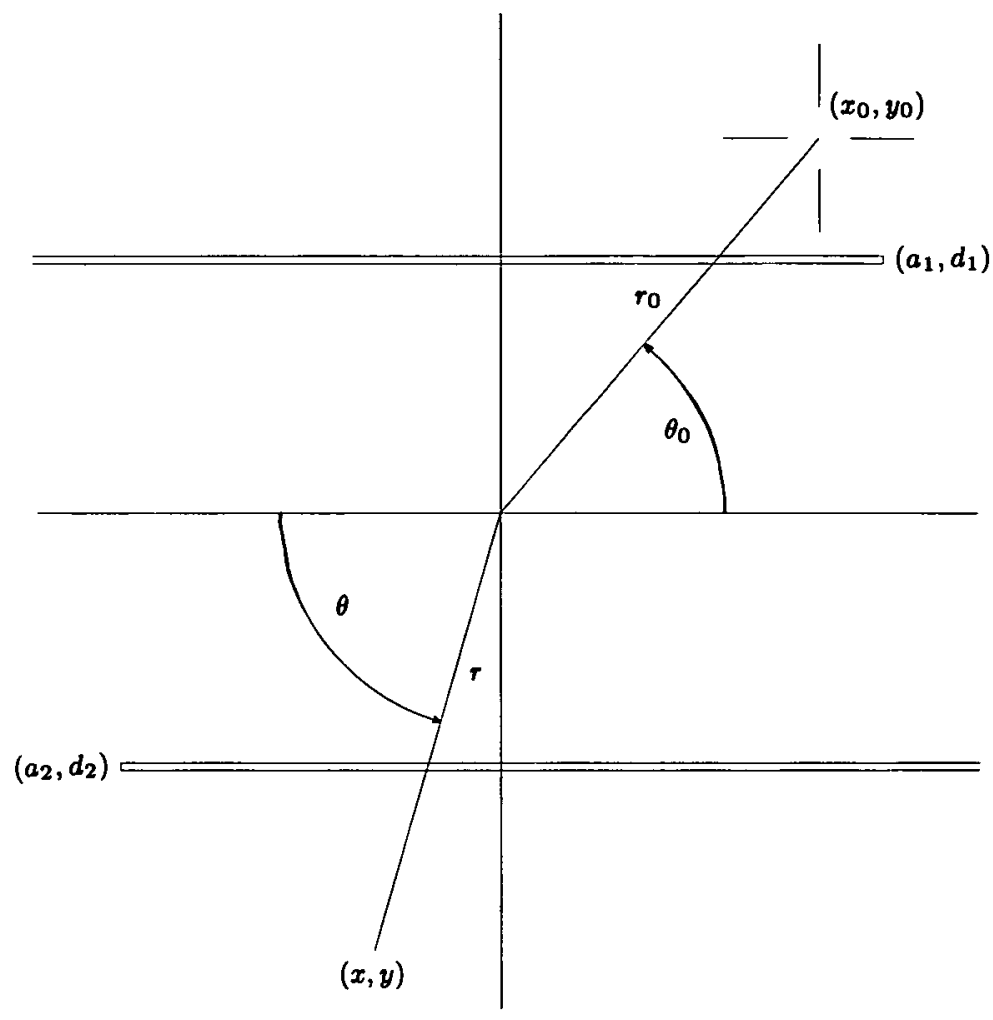

Figure 1. Source and field points and boundaries.

and $d_{1}>d_{2}$ will always apply. This area in the region $d_{2}<y<d_{1}$ and $a_{2}<x<a_{1}$ will be subsequently referred to as the duct. The Wiener-Hopf technique can be used to solve this problem but the Wiener-Hopf equations obtained are simultaneous, and the method suggested in Section 4.4 of [6] is employed.

When the ratio duct length divided by duct width is large, asymptotic solutions for the region on the opposite side to the source will be graphed to show the effect of a long thin duct on the field.

\section{Formulation of the problem}

Assume a time dependence of the form $\exp (-i \omega t)$ for the field, denoted by $\phi$, and consider a unit line source parallel to the $z$ axis at the point $\left(x_{0}, y_{0}\right)$, as in Figure 1. The wave equation reduces to Helmholtz's equation, with a source term independent of $z$. Thus, $\phi$ will be independent of $z$ and the problem reduces 
to a two-dimensional one. The equation for $\phi$ is:

$$
\frac{\partial^{2} \phi}{\partial x^{2}}+\frac{\partial^{2} \phi}{\partial y^{2}}+k^{2} \phi=-4 \pi \delta\left(x-x_{0}\right) \delta\left(y-y_{0}\right)
$$

where $\delta$ denotes the Dirac delta function and $k(=\omega / c, c$ is the speed of light) is the wavenumber. As is usual with problems of this sort, for analysis purposes assume that $k$ is a complex number with a small positive imaginary part as well as a real part. Once the problem has been solved, this small positive imaginary part can be let tend to zero. Also, consider Neumann boundary conditions, that is,

$$
\frac{\partial \phi}{\partial y}=0 \text { on } y=d_{1}, x<a_{1} \text { and } y=d_{2}, x>a_{2} .
$$

Edge conditions [2], [6] require that

$$
\phi=O(1)
$$

and

$$
\frac{\partial \phi}{\partial y}=O\left(r^{-1 / 2}\right)
$$

as $r \rightarrow 0$ where $r$ is the distance from either $\left(a_{1}, d_{1}\right)$ or $\left(a_{2}, d_{2}\right)$.

As discussed in Section 1, $\phi$ may be determined in the three regions $y<d_{2}$, $d_{2}<y<d_{1}$ and $y>d_{1}$ using, for example, Green's functions appropriate to each region, and these solutions linked across the boundaries. This approach is taken here. $\phi$ can be written, [5]:

$$
\phi=\left\{\begin{array}{c}
\pi i\left[H_{0}^{(1)}\left(k \sqrt{\left(x-x_{0}\right)^{2}+\left(y-y_{0}\right)^{2}}\right)\right. \\
\left.\quad+H_{0}^{(1)}\left(k \sqrt{\left(x-x_{0}\right)^{2}+\left(y+y_{0}-2 d_{1}\right)^{2}}\right)\right] \\
\quad+\frac{1}{2 i} \int_{-\infty}^{+\infty} f(\varsigma) H_{0}^{(1)}\left(k \sqrt{(x-\varsigma)^{2}+\left(y-d_{1}\right)^{2}}\right) d \varsigma \\
\quad\left(y>d_{1}\right) \\
\frac{i}{2\left(d_{1}-d_{2}\right)} \sum_{n=0}^{\infty}\left(\varepsilon_{n} / \beta_{n}\right) \cos \left(n \pi\left(y-d_{1}\right) /\left(d_{1}-d_{2}\right)\right) \int_{-\infty}^{+\infty} f(\varsigma) e^{i \beta_{n}|x-\varsigma|} d \varsigma \\
\frac{-i}{2\left(d_{1}-d_{2}\right)} \sum_{n=0}^{\infty}\left(\varepsilon_{n} / \beta_{n}\right) \cos \left(n \pi\left(y-d_{2}\right) /\left(d_{1}-d_{2}\right)\right) \int_{-\infty}^{+\infty} g(\gamma) e^{i \beta n|x-\gamma|} d \gamma \\
\frac{i}{2} \int_{-\infty}^{+\infty} g(\gamma) H_{0}^{(1)}\left(k \sqrt{(x-\gamma)^{2}+\left(y-d_{2}\right)^{2}}\right) d \gamma \\
\quad\left(y<d_{1}\right)
\end{array}\right.
$$

where

$$
\varepsilon_{n}= \begin{cases}1 & n=0 \\ 2 & n>0\end{cases}
$$


is the Neumann function, and

$$
\beta_{n}=\sqrt{k^{2}-n^{2} \pi^{2} /\left(d_{1}-d_{2}\right)^{2}},
$$

where the branch of the square root function is chosen such that $\operatorname{Im}\left(\beta_{n}\right)>0$ as $n \rightarrow \infty$. The boundary conditions (2.2), edge conditions (2.3) and (2.4) and the fact that $\phi$ must be continuous across $y=d_{1}, x<a_{1}$ and $y=d_{2}, x>a_{2}$ and $\partial \phi / \partial y$ continuous across $y=d_{1}, d_{2}$, allow for the determination of $f$ and $g$ in (2.5), as is done in Section 3. Note that equality of the derivative across $y=d_{1}$, $d_{2}$ in (2.5) already holds.

\section{Reduction to and solution by Wiener-Hopf technique}

Define

$$
\begin{gathered}
\Phi(\lambda, y)=\frac{1}{\sqrt{2 \pi}} \int_{-\infty}^{+\infty} \phi(x, y) e^{i \lambda x} d x \\
F(\lambda)=\frac{1}{\sqrt{2 \pi}} \int_{-\infty}^{+\infty} f(\zeta) e^{2 \lambda \zeta} d \zeta \\
G(\lambda)=\frac{1}{\sqrt{2 \pi}} \int_{-\infty}^{+\infty} g(\gamma) e^{i \lambda \gamma} d \gamma
\end{gathered}
$$

with inverses

$$
\begin{aligned}
\phi(x, y) & =\frac{1}{\sqrt{2 \pi}} \int_{-\infty}^{+\infty} \Phi(\lambda, y) e^{-i \lambda x} d \lambda, \\
f(\varsigma) & =\frac{1}{\sqrt{2 \pi}} \int_{-\infty}^{+\infty} F(\lambda) e^{-i \lambda \varsigma} d \lambda, \\
g(\gamma) & =\frac{1}{\sqrt{2 \pi}} \int_{-\infty}^{+\infty} G(\lambda) e^{-i \lambda \gamma} d \lambda .
\end{aligned}
$$

Take the Fourier Transform of (2.5) to get

$$
\Phi(\lambda, y)=\left\{\begin{array}{cc}
\sqrt{2 \pi} e^{i \lambda x_{0}}\left[e^{-\left|y-y_{0}\right| \sqrt{\lambda^{2}-k^{2}}}+e^{-\left(y_{0}+y-2 d_{1}\right) \sqrt{\lambda^{2}-k^{2}}}\right] / \sqrt{\lambda^{2}-k^{2}} \\
-F(\lambda) e^{-\left(y-d_{1}\right) \sqrt{\lambda^{2}-k^{2}}} / \sqrt{\lambda^{2}-k^{2}} \quad\left(y>d_{1}\right) \\
\frac{F(\lambda) \cosh \left[\left(y-d_{2}\right) \sqrt{\lambda^{2}-k^{2}}\right]-G(\lambda) \cosh \left[\left(y-d_{1}\right) \sqrt{\lambda^{2}-k^{2}}\right]}{\sqrt{\lambda^{2}-k^{2}} \sinh \left[\left(d_{1}-d_{2}\right) \sqrt{\lambda^{2}-k^{2}}\right]} \\
G(\lambda) e^{\left(y-d_{2}\right) \sqrt{\lambda^{2}-k^{2}}} / \sqrt{\lambda^{2}-k^{2}} \quad\left(y<d_{2}\right) .
\end{array}\right.
$$

From (3.7), we have,

$$
\left.\frac{\partial \Phi}{\partial y}\right|_{y=d_{1}}=F(\lambda),\left.\quad \frac{\partial \Phi}{\partial y}\right|_{y=d_{2}}=G(\lambda) .
$$




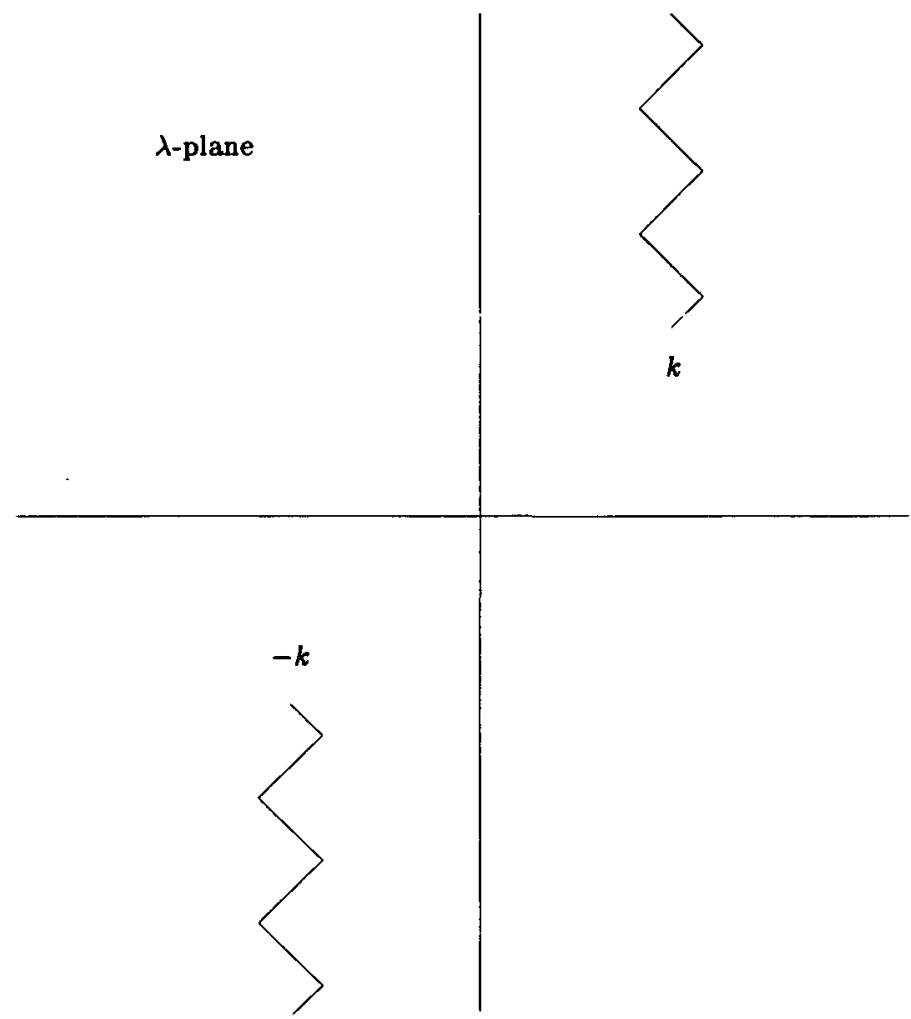

Figure 2. Branch cut system for $\lambda$ plane.

The function $\Phi(\lambda, y)$ defined in (3.7) has branch points at $\pm k$, and the usual branch-cut system, as illustrated in Figure 2, is used to satisfy the radiation condition (only outgoing waves at infinity). It is convenient at this time to define the upper half plane as that region for which $\operatorname{Im}(\lambda)>\operatorname{Im}(-k)$, and the lower half plane to be the region for which $\operatorname{Im}(\lambda)<\operatorname{Im}(k)$. Further, any function analytic in the upper half plane will be subscripted with a " + " and any function analytic in the lower half plane will be subscripted with a "-", as done by Noble [6].

Using this terminology, the two functions $F$ and $G$ may be split as follows,

$$
\begin{aligned}
F_{+}(\lambda) & =\frac{1}{\sqrt{2 \pi}} \int_{a_{1}}^{\infty} f(x) e^{i \lambda x} d x, \quad F_{-}(\lambda)=\frac{1}{\sqrt{2 \pi}} \int_{-\infty}^{a_{1}} f(x) e^{i \lambda x} d x \\
G_{+}(\lambda) & =\frac{1}{\sqrt{2 \pi}} \int_{a_{2}}^{\infty} g(x) e^{i \lambda x} d x, \quad G_{-}(\lambda)=\frac{1}{\sqrt{2 \pi}} \int_{-\infty}^{a_{2}} g(x) e^{i \lambda x} d x \\
F & =F_{+}+F_{-}, \quad G=G_{+}+G_{-}
\end{aligned}
$$


The boundary conditions (2.2) correspond to $F_{-}=0$ and $G_{+}=0$. Thus (3.7) becomes

$$
\Phi(\lambda, y)=\left\{\begin{array}{c}
\sqrt{2 \pi} e^{i \lambda x_{0}}\left[e^{-\left|y-y_{0}\right| \sqrt{\lambda^{2}-k^{2}}}+e^{-\left(y+y_{0}-2 d_{1}\right) \sqrt{\lambda^{2}-k^{2}}}\right] / \sqrt{\lambda^{2}-k^{2}} \\
-F_{+} e^{-\left(y-d_{1}\right) \sqrt{\lambda^{2}-k^{2}}} / \sqrt{\lambda^{2}-k^{2}} \\
\left(y>d_{1}\right) \\
\frac{F_{+} \cosh \left[\left(y-d_{2}\right) \sqrt{\lambda^{2}-k^{2}}\right]-G_{-} \cosh \left[\left(y-d_{1}\right) \sqrt{\lambda^{2}-k^{2}}\right]}{\sqrt{\lambda^{2}-k^{2}} \sinh \left[\left(d_{1}-d_{2}\right) \sqrt{\lambda^{2}-k^{2}}\right]} \\
\left(d_{2}<y<d_{1}\right) \\
G_{-} e^{\left(y-d_{2}\right) \sqrt{\lambda^{2}-k^{2}}} / \sqrt{\lambda^{2}-k^{2}} \quad\left(y<d_{2}\right)
\end{array}\right.
$$

The only condition left to enforce is the continuity of $\phi$ on $y=d_{1}, x>a_{1}$ and $y=d_{2}, x<a_{2}$. Define $\Phi_{1}$ and $\Phi_{2}$ for $\varepsilon$ small as

$$
\begin{aligned}
& \Phi_{1}^{+}\left(\lambda, d_{1} \pm \varepsilon\right)=\frac{1}{\sqrt{2 \pi}} \int_{a_{1}}^{\infty} \phi\left(x, d_{1} \pm \varepsilon\right) e^{i \lambda x} d x \\
& \Phi_{1}^{-}\left(\lambda, d_{1} \pm \varepsilon\right)=\frac{1}{\sqrt{2 \pi}} \int_{-\infty}^{a_{1}} \phi\left(x, d_{1} \pm \varepsilon\right) e^{i \lambda x} d x \\
& \Phi_{2}^{+}\left(\lambda, d_{2} \pm \varepsilon\right)=\frac{1}{\sqrt{2 \pi}} \int_{a_{2}}^{\infty} \phi\left(x, d_{2} \pm \varepsilon\right) e^{i \lambda x} d x \\
& \Phi_{2}^{-}\left(\lambda, d_{2} \pm \varepsilon\right)=\frac{1}{\sqrt{2 \pi}} \int_{-\infty}^{a_{2}} \phi\left(x, d_{2} \pm \varepsilon\right) e^{i \lambda x} d x
\end{aligned}
$$

Thus

$$
\begin{aligned}
\Phi\left(\lambda, d_{1}+\varepsilon\right)= & \Phi_{1}^{+}\left(\lambda, d_{1}+\varepsilon\right)+\Phi_{1}^{-}\left(\lambda, d_{1}+\varepsilon\right) \\
= & 2 \sqrt{2 \pi} e^{i \lambda x_{0}-\left(y_{0}-d_{1}\right) \sqrt{\lambda^{2}-k^{2}}} / \sqrt{\lambda^{2}-k^{2}} \\
& -F_{+} / \sqrt{\lambda^{2}-k^{2}} \\
\Phi\left(\lambda, d_{1}-\varepsilon\right)= & \Phi_{1}^{+}\left(\lambda, d_{1}-\varepsilon\right)+\Phi_{1}^{-}\left(\lambda, d_{1}-\varepsilon\right) \\
= & \frac{F_{+} \cosh \left[\left(d_{1}-d_{2}\right) \sqrt{\lambda^{2}-k^{2}}\right]-G_{-}}{\sqrt{\lambda^{2}-k^{2}} \sinh \left[\left(d_{1}-d_{2}\right) \sqrt{\lambda^{2}-k^{2}}\right]} \\
\Phi\left(\lambda, d_{2}+\varepsilon\right)= & \Phi_{2}^{+}\left(\lambda, d_{2}+\varepsilon\right)+\Phi_{2}^{-}\left(\lambda, d_{2}+\varepsilon\right) \\
= & \frac{F_{+}-G_{-} \cosh \left[\left(d_{1}-d_{2}\right) \sqrt{\lambda^{2}-k^{2}}\right]}{\sqrt{\lambda^{2}-k^{2}} \sinh \left[\left(d_{1}-d_{2}\right) \sqrt{\lambda^{2}-k^{2}}\right]} \\
\Phi\left(\lambda, d_{2}-\varepsilon\right)= & \Phi_{2}^{+}\left(\lambda, d_{2}-\varepsilon\right)+\Phi_{2}^{-}\left(\lambda, d_{2}-\varepsilon\right)=G_{-} / \sqrt{\lambda^{2}-k^{2}}
\end{aligned}
$$

as $\varepsilon \rightarrow 0^{+}$. As $\phi$ is continuous across $y=d_{1}, x>a_{1}$, and $y=d_{2}, x<a_{2}$,

$$
\begin{aligned}
& \Phi_{1}^{+}\left(\lambda, d_{1}+\varepsilon\right)=\Phi_{1}^{+}\left(\lambda, d_{1}-\varepsilon\right), \\
& \Phi_{2}^{-}\left(\lambda, d_{2}+\varepsilon\right)=\Phi_{2}^{-}\left(\lambda, d_{2}-\varepsilon\right) .
\end{aligned}
$$


Define

$$
\begin{aligned}
& D_{1}^{-}=\Phi_{1}^{-}\left(\lambda, d_{1}+\varepsilon\right)-\Phi_{1}^{-}\left(\lambda, d_{1}-\varepsilon\right), \\
& D_{2}^{+}=\Phi_{2}^{+}\left(\lambda, d_{2}+\varepsilon\right)-\Phi_{2}^{+}\left(\lambda, d_{2}-\varepsilon\right) .
\end{aligned}
$$

Subtract (3.16) from (3.15) and (3.18) from (3.17) to get

$$
\begin{aligned}
& D_{1}^{-}=\frac{2 \sqrt{2 \pi} e^{i \lambda x_{0}-\left(y_{0}-d_{1}\right) \sqrt{\lambda^{2}-k^{2}}}}{\sqrt{\lambda^{2}-k^{2}}}-\frac{F_{+} e^{\left(d_{1}-d_{2}\right) \sqrt{\lambda^{2}-k^{2}}}-G_{-}}{\sqrt{\lambda^{2}-k^{2}} \sinh \left[\left(d_{1}-d_{2}\right) \sqrt{\lambda^{2}-k^{2}}\right]}, \\
& D_{2}^{+}=\frac{F_{+}-G_{-} e^{\left(d_{1}-d_{2}\right) \sqrt{\lambda^{2}-k^{2}}}}{\sqrt{\lambda^{2}-k^{2}} \sinh \left[\left(d_{1}-d_{2}\right) \sqrt{\lambda^{2}-k^{2}}\right]} .
\end{aligned}
$$

Equations (3.23) and (3.24) are the dual Wiener-Hopf equations. A quick check, involving writing (3.23) and (3.24) out in matrix form, ensures that the method due to R. A. Hurd [1] cannot be used here, as the resulting ' $G$ ' matrix referred to in equation (3) of his paper does not factor into the appropriate form. To ensure uniqueness, edge conditions (2.3) and (2.4) give

$$
\begin{aligned}
& F_{+} \sim e^{i \lambda a_{1}}|\lambda|^{-1 / 2}, \\
& G_{-} \sim e^{i \lambda a_{2}}|\lambda|^{-1 / 2}, \\
& D_{1}^{-} \sim e^{i \lambda a_{1}}|\lambda|^{-1}, \\
& D_{2}^{+} \sim e^{i \lambda a_{2}}|\lambda|^{-1}
\end{aligned}
$$

as $\lambda \rightarrow \infty$ in the appropriate half planes. Define

$$
M_{+}(\lambda) M_{-}(\lambda)=e^{-\left(d_{1}-d_{2}\right) \sqrt{\lambda^{2}-k^{2}}}\left(d_{1}-d_{2}\right) \sqrt{\lambda^{2}-k^{2}} \sinh \left[\left(d_{1}-d_{2}\right) \sqrt{\lambda^{2}-k^{2}}\right]
$$

where $M_{+}$and $M_{-}$are asymptotic to $|\lambda|^{1 / 2}$ in appropriate half planes. Thus,

$$
\begin{aligned}
& M_{ \pm}(\lambda)=\left(d_{1}-d_{2}\right)(\lambda \pm k) \\
& \quad \times \exp \left\{ \pm i \lambda\left(d_{1}-d_{2}\right)\left[1-C+\ln \left(2 \pi / k\left(d_{1}-d_{2}\right)\right)\right] / \pi \mp \lambda\left(d_{1}-d_{2}\right) / 2\right\} \\
& \quad \times \exp \left\{-\left(d_{1}-d_{2}\right) \sqrt{\lambda^{2}-k^{2}} \arccos ( \pm \lambda / k) / \pi\right\} \\
& \quad \times \prod_{n=1}^{\infty}\left[\left(1-k^{2}\left(d_{1}-d_{2}\right)^{2} / n^{2} \pi^{2}\right)^{1 / 2} \mp i \lambda\left(d_{1}-d_{2}\right) / n \pi\right] \exp \left\{i \lambda\left(d_{1}-d_{2}\right) / n \pi\right\}
\end{aligned}
$$

where $C$ is Euler's constant $=0.5772 \cdots$. The functions $M_{+}$and $M_{-}$are related to Noble's functions $L_{+}$and $L_{-}$in [3] by

$$
M_{ \pm}(\lambda)=\left(d_{1}-d_{2}\right)(\lambda \pm k) L_{ \pm}(\lambda)
$$


Equation (3.23) becomes

$$
\begin{aligned}
\frac{D_{1}^{-} M_{-} e^{-i \lambda a_{1}}}{\left(d_{1}-d_{2}\right)}= & \frac{2 \sqrt{2 \pi} e^{i \lambda\left(x_{0}-a_{1}\right)-\left(y_{0}-d_{1}\right) \sqrt{\lambda^{2}-k^{2}}} M_{-}}{\left(d_{1}-d_{2}\right) \sqrt{\lambda^{2}-k^{2}}} \\
& -\frac{F_{+} e^{-i \lambda a_{1}}}{M_{+}}+\frac{G_{-} M_{-} e^{-i \lambda a_{1}}}{\left(d_{1}-d_{2}\right) \sqrt{\lambda^{2}-k^{2}} \sinh \left[\left(d_{1}-d_{2}\right) \sqrt{\lambda^{2}-k^{2}}\right.} .
\end{aligned}
$$

It is at this point that this derivation differs from the work by Kashyap [4], who deals with the same problem but with $\left(a_{1}-a_{2}\right)<0$. It is necessary here to multiply (3.23) by $\exp \left(-i \lambda a_{1}\right)$ to ensure bounded behaviour of all functions as $\lambda \rightarrow \infty$ in the appropriate half planes. Thus, the last term in (3.23) and subsequent infinite series expansion is really introduced as a consequence of this.

Now,

$$
\begin{aligned}
& \frac{1}{\left(d_{1}-d_{2}\right) \sqrt{\lambda^{2}-k^{2}} \sinh \left[\left(d_{1}-d_{2}\right) \sqrt{\lambda^{2}-k^{2}}\right]} \\
& =\frac{-1}{2\left(d_{1}-d_{2}\right)^{2}} \sum_{n=0}^{\infty} \frac{\varepsilon_{n}(-1)^{n}}{\beta_{n}\left(\lambda+\beta_{n}\right)}+\frac{1}{2\left(d_{1}-d_{2}\right)^{2}} \sum_{n=0}^{\infty} \frac{\varepsilon_{n}(-1)^{n}}{\beta_{n}\left(\lambda-\beta_{n}\right)},
\end{aligned}
$$

where the first function on the right-hand side of (3.30) is analytic in the upper half-plane and the second function is analytic in the lower half-plane. By splitting the term with sinh in the denominator up into terms analytic in the upper and lower half planes, (3.29) becomes

$$
\begin{aligned}
& \frac{D_{1}^{-} M_{-} e^{-i \lambda a_{1}}}{\left(d_{1}-d_{2}\right)}=\frac{2 \sqrt{2 \pi} e^{i \lambda\left(x_{0}-a_{1}\right)-\left(y_{0}-d_{1}\right) \sqrt{\lambda^{2}-k^{2}}} M_{-}}{\left(d_{1}-d_{2}\right) \sqrt{\lambda^{2}-k^{2}}} \\
& -F_{+} e^{-i \lambda a_{1}} / M_{+}+G_{-} M_{-} e^{-i \lambda a_{1}} b_{-} \\
& -\frac{1}{2\left(d_{1}-d_{2}\right)^{2}} \sum_{n=0}^{\infty} \frac{\varepsilon_{n}(-1)^{n} G_{-}\left(-\beta_{n}\right) M_{-}\left(-\beta_{n}\right) e^{i a_{1} \beta_{n}}}{\beta_{n}\left(\lambda+\beta_{n}\right)} \\
& -\frac{1}{2\left(d_{1}-d_{2}\right)^{2}} \sum_{n=0}^{\infty} \frac{\varepsilon_{n}(-1)^{n}\left[G_{-} M_{-} e^{-i \lambda a_{1}}-G_{-}\left(-\beta_{n}\right) M_{-}\left(-\beta_{n}\right) e^{i a_{1} \beta_{n}}\right]}{\beta_{n}\left(\lambda+\beta_{n}\right)}
\end{aligned}
$$

where

$$
\begin{aligned}
& b_{+}(\lambda)=\frac{-1}{2\left(d_{1}-d_{2}\right)^{2}} \sum_{n=0}^{\infty} \frac{\varepsilon_{n}(-1)^{n}}{\beta_{n}\left(\lambda+\beta_{n}\right)}, \\
& b_{-}(\lambda)=\frac{1}{2\left(d_{1}-d_{2}\right)^{2}} \sum_{n=0}^{\infty} \frac{\varepsilon_{n}(-1)^{n}}{\beta_{n}\left(\lambda-\beta_{n}\right)} .
\end{aligned}
$$

The source term can be split (refer Noble [6]) as follows:

$$
\frac{2 \sqrt{2 \pi} e^{i \lambda\left(x_{0}-a_{1}\right)-\left(y_{0}-d_{1}\right) \sqrt{\lambda^{2}-k^{2}}}}{\left(d_{1}-d_{2}\right) \sqrt{\lambda^{2}-k^{2}}}=h_{+}(\lambda)+h_{-}(\lambda)
$$


where

$$
\begin{aligned}
& h_{+}(\lambda)=\frac{1}{2 \pi i} \int_{-\infty}^{+\infty} \frac{2 \sqrt{2 \pi} e^{2 \zeta\left(x_{0}-a_{1}\right)-\left(y_{0}-d_{1}\right) \sqrt{\varsigma^{2}-k^{2}}} M_{-}(\varsigma) d \zeta}{\left(d_{1}-d_{2}\right) \sqrt{\varsigma^{2}-k^{2}}(\varsigma-\lambda)} \\
& \text { for } \operatorname{Im}(-k)<\operatorname{Im}(\varsigma)<\operatorname{Im}(\lambda)<\operatorname{Im}(k) \text {, } \\
& h_{-}(\lambda)=\frac{-1}{2 \pi i} \int_{-\infty}^{+\infty} \frac{2 \sqrt{2 \pi} e^{i \zeta\left(x_{0}-a_{1}\right)-\left(y_{0}-d_{1}\right) \sqrt{\varsigma^{2}-k^{2}}} M_{-}(\varsigma) d \varsigma}{\left(d_{1}-d_{2}\right) \sqrt{\zeta^{2}-h^{2}}(\zeta-\lambda)} \\
& \text { for } \operatorname{Im}(-k)<\operatorname{Im}(\lambda)<\operatorname{Im}(\varsigma)<\operatorname{Im}(k) \text {. }
\end{aligned}
$$

Thus,

$$
\begin{aligned}
h_{+}- & \frac{F_{+} e^{-i \lambda a_{1}}}{M_{+}}-\frac{1}{2\left(d_{1}-d_{2}\right)^{2}} \sum_{n=0}^{\infty} \frac{\varepsilon_{n}(-1)^{n} G_{-}\left(-\beta_{n}\right) M_{-}\left(-\beta_{n}\right) e^{i a_{1} \beta_{n}}}{\beta_{n}\left(\lambda+\beta_{n}\right)} \\
= & \frac{D_{1}^{-} M_{-} e^{-i \lambda a_{1}}}{\left(d_{1}-d_{2}\right)}-h_{-}-G_{-} M_{-} b_{-} e^{-i \lambda a_{1}} \\
& +\frac{1}{2\left(d_{1}-d_{2}\right)^{2}} \sum_{n=0}^{\infty} \frac{\varepsilon_{n}(-1)^{n}\left[G_{-} M_{-} e^{-i \lambda a_{1}}-G_{-}\left(-\beta_{n}\right) M_{-}\left(-\beta_{n}\right) e^{i a_{1} \beta_{n}}\right]}{\beta_{n}\left(\lambda+\beta_{n}\right)} .
\end{aligned}
$$

Similar analysis on (3.24) gives

$$
\begin{aligned}
& \frac{D_{2}^{+} M_{+} e^{-i \lambda a_{2}}}{\left(d_{1}-d_{2}\right)}-F_{+} M_{+} b_{+} e^{-i \lambda a_{2}} \\
& -\frac{1}{2\left(d_{1}-d_{2}\right)^{2}} \sum_{n=0}^{\infty} \frac{\varepsilon_{n}(-1)^{n}\left[F_{+} M_{+} e^{-i \lambda a_{2}}-F_{+}\left(\beta_{n}\right) M_{+}\left(\beta_{n}\right) e^{-i a_{2} \beta_{n}}\right]}{\beta_{n}\left(\lambda-\beta_{n}\right)} \\
& \quad=\frac{1}{2\left(d_{1}-d_{2}\right)^{2}} \sum_{n=0}^{\infty} \frac{\varepsilon_{n}(-1)^{n} F_{+}\left(\beta_{n}\right) M_{+}\left(\beta_{n}\right) e^{-i a_{2} \beta_{n}}}{\beta_{n}\left(\lambda-\beta_{n}\right)}-\frac{G_{-} e^{-i \lambda a_{2}}}{M_{-}} .
\end{aligned}
$$

Note that the series in (3.37) and (3.38) can be shown to be absolutely convergent, except near $\lambda=\beta_{m}, m=0,1,2, \ldots$, by using (3.25). The left-hand sides of (3.37) and (3.38) are analytic in the upper half plane, and the righthand sides are analytic in the lower half plane. Equations (3.37) and (3.38), in conjunction with (3.25), imply that both sides of both equations are $O\left(\lambda^{-1}\right)$ as $\lambda \rightarrow \infty$ in the appropriate half-planes. It follows from Liouville's theorem that the left and right hand sides of (3.37) and (3.38) are analytic representations, with the overlapping domain $\operatorname{Im}(-k)<\operatorname{Im}(\lambda)<\operatorname{Im}(k)$, of the integral function 0 .

To solve for $F_{+}\left(\beta_{m}\right)$ and $G_{-}\left(-\beta_{m}\right)$, set $\lambda=\beta_{m}$ in the right-hand side of (3.37) and $\lambda=-\beta_{m}$ in the right-hand side of (3.38). This gives an infinite set of coupled equations for $m=1,2, \ldots$. Set

$$
\begin{aligned}
& p_{m}=F_{+}\left(\beta_{m}\right) e^{-i a_{1} \beta_{m}}, \\
& q_{m}=G_{-}\left(-\beta_{m}\right) e^{i a_{2} \beta_{m}}
\end{aligned}
$$


where, using (3.25), $p_{m}, q_{m} \sim m^{-1 / 2}$ as $m \rightarrow \infty$. Thus the equations for $p_{m}$ and $q_{m}$ are

$$
\begin{aligned}
& p_{m}+\frac{M_{+}\left(\beta_{m}\right)}{2\left(d_{1}-d_{2}\right)^{2}} \sum_{n=0}^{\infty} \frac{\varepsilon_{n}(-1)^{n} M_{-}\left(-\beta_{n}\right) e^{i\left(a_{1}-a_{2}\right) \beta_{n}} q_{n}}{\beta_{n}\left(\beta_{m}+\beta_{n}\right)}=M_{+}\left(\beta_{m}\right) h_{+}\left(\beta_{m}\right), \\
& q_{m}+\frac{M_{-}\left(-\beta_{m}\right)}{2\left(d_{1}-d_{2}\right)^{2}} \sum_{n=0}^{\infty} \frac{\varepsilon_{n}(-1)^{n} M_{+}\left(\beta_{n}\right) e^{i\left(a_{1}-a_{2}\right) \beta_{n}} p_{n}}{\beta_{n}\left(\beta_{m}+\beta_{n}\right)}=0
\end{aligned}
$$

\section{Asymptotic form for waves below first cutoff}

As an example, consider the case $2<k<20,\left(d_{1}-d_{2}\right)=0.002$ and $\left(a_{1}-a_{2}\right)=$ 0.30. A method similar to that used by D. S. Jones [3] can be used to find an approximate solution for the asymptotic expansion for this example. The expression for $G_{-}$in (3.38), substituted into (3.7) and noting (3.39), gives

$$
\begin{gathered}
\Phi(\lambda, y)=\frac{1}{2\left(d_{1} d_{2}\right)^{2}} \sum_{n=0}^{\infty} \frac{\varepsilon_{n}(-1)^{n} M_{+}\left(\beta_{n}\right) e^{i\left(a_{1}-a_{2}\right) \beta_{n}} p_{n}}{\beta_{n}\left(\lambda-\beta_{n}\right)} \frac{M_{-} e^{i \lambda a_{2}+\left(y-d_{2}\right) \sqrt{\lambda^{2}-k^{2}}}}{\sqrt{\lambda^{2}-k^{2}}} \\
\text { for } y<d_{2} .
\end{gathered}
$$

Thus,

$$
\begin{aligned}
\phi(x, y)= & \frac{1}{\sqrt{2 \pi} 2\left(d_{1}-d_{2}\right)^{2}} \sum_{n=0}^{\infty} \frac{\varepsilon_{n}(-1)^{n} M_{+}\left(\beta_{n}\right) e^{i\left(a_{1}-a_{2}\right) \beta_{n}} p_{n}}{\beta_{n}} \\
& \times \int_{-\infty}^{+\infty} \frac{M_{-}(\lambda) e^{-i \lambda\left(x-a_{2}\right)+\left(y-d_{2}\right) \sqrt{\lambda^{2}-k^{2}}} d \lambda}{\sqrt{\lambda^{2}-k^{2}}\left(\lambda-\beta_{n}\right)} \text { for } y<d_{2} .
\end{aligned}
$$

For $k,\left(d_{1}-d_{2}\right)$ and $\left(a_{1}-a_{2}\right)$ as above, $\beta_{0}=k$ and for $n>0, \operatorname{Re}\left(\beta_{n}\right)=0$ and $\operatorname{Im}\left(\left(a_{1}-a_{2}\right) \beta_{n}\right)>100 . n$. Thus, $\exp \left\{i\left(a_{1}-a_{2}\right) \beta_{n}\right\} \ll 1$ for $n>0$ and (3.41) and (3.42) can be solved accordingly. Set

$$
\begin{aligned}
& p_{m}=P_{m}\left(1+O\left(\exp \left\{-\pi\left(a_{1}-a_{2}\right) /\left(d_{1}-d_{2}\right)\right\}\right)\right), \\
& q_{m}=Q_{m}\left(1+O\left(\exp \left\{-\pi\left(a_{1}-a_{2}\right) /\left(d_{1}-d_{2}\right)\right\}\right)\right)
\end{aligned}
$$

in (3.41), (3.42). Thus,

$$
\begin{aligned}
& P_{m}+\frac{M_{+}\left(\beta_{m}\right) e^{i k\left(a_{1}-a_{2}\right)} M_{-}\left(-\beta_{0}\right) Q_{0}}{2\left(d_{1}-d_{2}\right)^{2} \beta_{0}\left(\beta_{m}+\beta_{0}\right)}=M_{+}\left(\beta_{m}\right) h_{+}\left(\beta_{m}\right) \\
& Q_{m}+\frac{M_{-}\left(-\beta_{m}\right) e^{i k\left(a_{1}-a_{2}\right)} M_{+}\left(\beta_{0}\right) P_{0}}{2\left(d_{1}-d_{2}\right)^{2} \beta_{0}\left(\beta_{m}+\beta_{0}\right)}=0 .
\end{aligned}
$$


Solve for $P_{0}, Q_{0}$ and $P_{m}, m>0$, to get

$$
\begin{aligned}
P_{0}= & \frac{16\left(d_{1}-d_{2}\right)^{4} \beta_{0}^{4} M_{+}\left(\beta_{0}\right) h_{+}\left(\beta_{0}\right)}{16 \beta_{0}^{4}\left(d_{1}-d_{2}\right)^{4}-M_{+}^{2}\left(\beta_{0}\right) M_{-}^{2}\left(-\beta_{0}\right) \exp \left\{i 2 k\left(a_{1}-a_{2}\right)\right\}} \\
Q_{0}= & \frac{4\left(d_{1}-d_{2}\right)^{2} \beta_{0}^{2} M_{-}\left(-\beta_{0}\right) M_{+}^{2}\left(\beta_{0}\right) h_{+}\left(\beta_{0}\right)}{M_{+}^{2}\left(\beta_{0}\right) M_{-}^{2}\left(-\beta_{0}\right) \exp \left\{i 2 k\left(a_{1}-a_{2}\right)\right\}-16 \beta_{0}^{4}\left(d_{1}-d_{2}\right)^{4}} \\
P_{m}= & M_{+}\left(\beta_{m}\right) h_{+}\left(\beta_{m}\right) \\
& -\frac{2 M_{+}\left(\beta_{m}\right) \beta_{0} M_{-}^{2}\left(-\beta_{0}\right) M_{+}^{2}\left(\beta_{0}\right) h_{+}\left(\beta_{0}\right) e^{i k\left(a_{1}-a_{2}\right)}}{\left(\beta_{M}+\beta_{0}\right)\left[M_{+}^{2}\left(\beta_{0}\right) M_{-}^{2}\left(-\beta_{0}\right) \exp \left\{i 2 k\left(a_{1}-a_{2}\right)\right\}-16 \beta_{0}^{4}\left(d_{1}-d_{2}\right)^{4}\right]}
\end{aligned}
$$

These substituted into (4.2) and neglecting terms of $O\left(\exp \left\{-\pi\left(a_{1}-a_{2}\right) /\left(d_{1}-\right.\right.\right.$ $\left.\left.d_{2}\right)\right\}$ ) noting (3.35) gives

$$
\begin{aligned}
& \phi(x, y)= \frac{8 k^{3}\left(d_{1}-d_{2}\right) M_{+}^{2}(k) \exp \left\{i k\left(a_{1}-a_{2}\right)\right\}}{\pi i\left[16 k^{4}\left(d_{1}-d_{2}\right)^{4}-M_{+}^{2}(k) M_{-}^{2}(-k) \exp \left\{i 2 k\left(a_{1}-a_{2}\right)\right\}\right]} \\
& \times \int_{-\infty}^{+\infty} \frac{\exp \left\{i \varsigma\left(x_{0}-a_{1}\right)-\left(y_{0}-d_{1}\right) \sqrt{\varsigma^{2}-k^{2}}\right\} M_{-}(\varsigma)}{\sqrt{\varsigma^{2}-k^{2}}(\varsigma-k)} d \varsigma \\
& \times \int_{-\infty}^{+\infty} \frac{\exp \left\{i \lambda\left(x-a_{2}\right)+\left(y-d_{2}\right) \sqrt{\lambda^{2}-k^{2}}\right\} M_{-}(\lambda)}{\sqrt{\lambda^{2}-k^{2}}(\lambda-k)} d \lambda \\
& \cdot\left\{1+O\left(\exp \left\{-\pi\left(a_{1}-a_{2}\right) /\left(d_{1}-d_{2}\right)\right\}\right)\right\} \\
& \text { for } y<d_{2} .
\end{aligned}
$$

Further, let

$$
\begin{array}{ll}
x_{0}=r_{0} \cos \theta_{0}, & x=r \cos (\theta+\pi), \\
y_{0}=r_{0} \sin \theta_{0}, & y=r \sin (\theta+\pi) \quad 0 \leq \theta, \theta_{0} \leq \pi
\end{array}
$$

as in Figure 1 and consider $r_{0}, r \rightarrow \infty$. After some standard analysis as per Noble [6], and noting this formula,

$$
\begin{aligned}
& \int_{-\infty}^{+\infty} f(\lambda) \exp \left\{-i \lambda x-|y| \sqrt{\lambda^{2}-k^{2}}\right\} d \lambda \\
& \sim(2 k \pi)^{1 / 2} \exp (-i \pi / 4) f(-k \cos \theta) \sin \theta r^{-1 / 2} \exp (i k r) \\
& \text { for } r \text { large }
\end{aligned}
$$

(4.10) becomes

$$
\begin{gathered}
\phi \sim \frac{16 M_{+}^{2}(k) M_{-}(-k \cos \theta) M_{-}\left(-k \cos \theta_{0}\right)\left(d_{1}-d_{2}\right)}{\left[M_{+}^{2}(k) M_{-}^{2}(-k) e^{i 2 k\left(a_{1}-a_{2}\right)}-16 k^{4}\left(d_{1}-d_{2}\right)^{4}\right](1+\cos \theta)\left(1+\cos \theta_{0}\right)\left(r r_{0}\right)^{1 / 2}} \\
\quad \times \exp \left\{i k\left(r_{0}+r+a_{1}-a_{2}+a_{1} \cos \theta_{0}-a_{2} \cos \theta-d_{1} \sin \theta_{0}+d_{2} \sin \theta\right)\right\} \\
\text { for } r, r_{0} \text { large }
\end{gathered}
$$




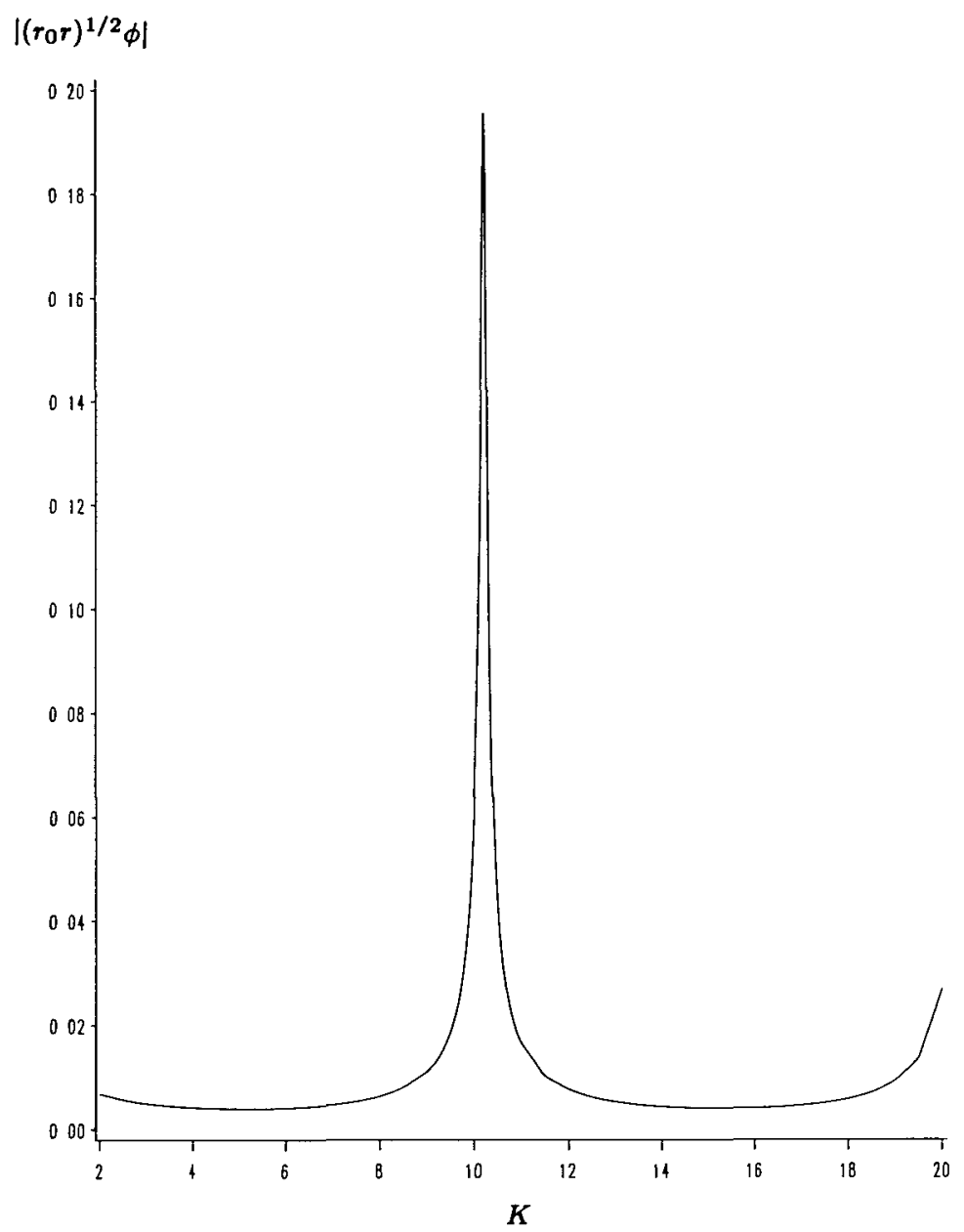

Figure 3. $\left|\left(r_{0} r\right)^{1 / 2} \phi\right|$ vs $k$ for $r_{0}$ and $r$ large.

Figure 3 shows the behaviour of $\left|\left(r_{0} r\right)^{1 / 2} \phi\right|$ with $\theta=\theta_{0}=\pi / 2$, for both $r_{0}$ and $r$ large enough to warrant using the asymptotic expansion but otherwise unspecified. This fact, of course, needs to be borne in mind when reading the vertical scale of the graph.

As can be seen from Figure 3, there is a marked peak for the far field at around $k=10.5$. This is because the duct acts as a waveguide. The field in the duct may be found from the inversion integral (3.4) with $\Phi$ given by (3.7), noting that $d_{2}<y<d_{1}$ in the duct. For $a_{2}<x<a_{1}$, all singularities are simple poles and the first mode in the duct, which will be the sum of two waves of the form $\exp \left\{-i k\left(x-a_{1}\right)\right\}$ and $\exp \left\{i k\left(x-a_{2}\right)\right\}$, will be dominant as all other modes 
are $O\left(\exp \left\{-\pi\left(a_{1}-a_{2}\right) /\left(d_{1}-d_{2}\right)\right\}\right)$ in comparison. As in standard waveguide theory, resonances will be established with standing waves in the duct when $k\left(a_{1}-a_{2}\right)=n . \pi$. For the value $a_{1}-a_{2}=0.3$, resonances can be expected at $k=10.5,21.0$, etc., which is consistent with Figure 3. The far-field strength is thus closely coupled to the tuning of the source to the resonancy conditions of the duct.

Note that the plane wave approximation may be derived by multiplying all fields by the factor

$$
\sqrt{\pi k r_{0} / 2} \exp \left\{-i k r_{0}+i \pi / 4\right\}
$$

and letting $r_{0} \rightarrow \infty$.

\section{Acknowledgements}

I should like to thank Dr Ian Doherty of the Department of Defence for many constructive suggestions and discussions, and the referees.

\section{References}

[1] R. A. Hurd, "A note on the solvability of simultaneous Wiener-Hopf equations", Canad. J. Phys. 57 (1979), 402-403.

[2] D. S. Jones, Theory of electromagnetism (Permagon Press, 1964).

[3] D. S. Jones, "Diffraction by a thick semi-infinite plate", Proc. Roy. Soc. London Ser. A 217 (1953), 153-175.

[4] S. C. Kashyap, "Diffraction characteristics of a slit formed by two staggered planes", $J$. Math. Phys. 15 (1974), 1944-1949.

[5] P. M. Morse and H. Feshbach, Methods of theoretical physics- Part I (McGraw-Hill, 1953).

[6] B. Noble, Methods based on the Wiener Hopf technique for the solution of partial differential equations (Permagon Press, 1958). 